\title{
Integrated plasma physics modelling for the Culham steady state spherical tokamak fusion power plant
}

\author{
H.R. Wilson, J.-W. Ahn ${ }^{1}$, R.J. Akers, D. Applegate ${ }^{1}$, R.A. Cairns ${ }^{2}$, \\ J.P. Christiansen, J.W. Connor, G. Counsell, A. Dnestrovskij ${ }^{3}$, \\ W.D. Dorland ${ }^{4}$, M.J. Hole ${ }^{5}$, N. Joiner ${ }^{1}$, A. Kirk, P.J. Knight, \\ C.N. Lashmore-Davies, K.G. McClements, D.E. McGregor ${ }^{2}$, \\ M.R. O'Brien, C.M. Roach, S. Tsaun ${ }^{3}$ and G.M. Voss \\ EURATOM/UKAEA Fusion Association, Culham Science Centre, Abingdon, Oxon OX14 \\ 3DB, UK \\ ${ }^{1}$ Physics Department, Imperial College, Prince Consort Road, London, SW7 2BZ, UK \\ ${ }^{2}$ University of St Andrews, Fife, KY16 9YSS, UK \\ ${ }^{3}$ Russian Science Centre, Kurchatov Institute, 123182 Kurchatov Square 1, Moscow, Russia \\ ${ }^{4}$ Department of Physics, University of Maryland, 3325 A V Williams Building, \\ College Park, Maryland 20742, USA \\ ${ }^{5}$ University of Sydney, Sydney, NSW 2006, Australia
}

Received 21 January 2004, accepted for publication 11 June 2004

Published 20 July 2004

Online at stacks.iop.org/NF/44/917

doi:10.1088/0029-5515/44/8/010

\begin{abstract}
Integrated modelling of important plasma physics issues related to the design of a steady-state spherical tokamak (ST) fusion power plant is described. The key is a steady-state current drive, and $92 \%$ of this is provided by a combination of bootstrap and diamagnetic currents, both of which have a substantial toroidal component in a ST. The remaining current is to be provided by either neutral beam injection or radio-frequency waves, and various schemes for providing this are discussed and quantified. The desire to achieve a high bootstrap current drives the design to high plasma pressure, $\beta$ (normalized to the magnetic field pressure), and high elongation. Both these requirements have implications for ideal magneto-hydrodynamic instability which are discussed. Confinement is addressed both through comparison with the recent scaling laws developed from the conventional tokamak database and selfconsistent one-dimensional modelling of the transport processes. This modelling shows that the power required for the current drive $(\sim 50 \mathrm{MW})$ is sufficient to heat the plasma to a regime where more than $3 \mathrm{GW}$ of fusion power is produced, taking into account the dilution due to He ash and prompt $\alpha$-particle losses, which are small. A preliminary study of the micro-instabilities, which may be responsible for the turbulent transport is provided. Given assumptions about the particle confinement, we make estimates of the fuelling requirements to maintain the steady state. Finally, the power loading due to the exhaust is derived using theory-based scalings for the scrape-off layer width.
\end{abstract}

PACS numbers: 28.52.Av

(Some figures in this article are in colour only in the electronic version)

\section{Introduction}

Demonstration of steady-state operation of a tokamak requires a self-consistent solution for the equilibrium, confinement, current drive and exhaust. This is difficult as they are all interlinked, particularly when the fusion power provides the dominant heating, as anticipated for a fusion power plant. For example, consider the influence of the plasma density. On the one hand, we wish to operate at relatively high density in order to improve confinement and fusion power but, on the other hand, the current drive requirements (e.g. required power from a neutral beam injection (NBI) system) are minimized at low density. High $\beta$ operation is also desirable, both to minimize Ohmic heating losses in the toroidal field circuit and also to maximize the bootstrap current: it is then necessary to take account of the magneto-hydrodynamic (MHD) stability limits. High elongation is beneficial for both confinement and the bootstrap current, but one then needs to address the 
vertical stability limits and any need for vertical feedback control systems. Optimizing such a highly non-linear system is a formidable task, and it is not even clear at the outset that a self-consistent solution exists.

In this paper, we concentrate on demonstrating that a selfconsistent solution for the plasma element of a steady-state ST fusion power plant [1] does indeed exist. The earliest studies demonstrated such a solution based primarily on systems codes, which employ simplified physics and engineering models to allow the non-linear system to be optimized [2-4]. Useful insight into the output from these codes has been provided by analytical models [5]. More recently, both the plasma physics and the engineering aspects of various fusion power plant designs have been studied in more detail and these confirm that the spherical tokamak (ST) could indeed provide a promising solution for a steady-state fusion power plant [6-11]. In this paper, we focus on one particular design for which the engineering basis is relatively well advanced, and explore the plasma physics in more detail. The main purposes of this paper are to: update the earlier plasma physics studies $[12,13]$, to re-assess the potential for the ST to provide fusion power, and to identify key topics for future research which would help confirm this design. The emphasis will be on predictions based on theoretical modelling but, where relevant, use will be made of the data from the MAST ST $[14,15]$

This paper is set out as follows. In the next section we review the basic principles which guide the design of a steadystate ST power plant (STPP), and describe the main parameters of the design we choose to study. Then, in section 3 , we address a number of plasma physics issues related to the key topics of confinement, stability, heating and current drive, fuelling and exhaust. The emphasis here is on demonstrating that a selfconsistent solution does exist, so we shall concentrate mainly on the properties of the machine parameters of the design described in section 2, rather than present parameter scans. A discussion and conclusions are provided in section 4 .

\section{Design philosophy}

In this section we describe how we arrive at our chosen design, the main parameters of which are summarized in table 1 . Although for the sake of clear presentation we describe our design as if it is driven mainly by the engineering requirements, with the plasma physics added in at the end, this is not the case. Rather, the design has been developed over many years, drawing on experience from systems code results (which fully integrate engineering constraints and plasma physics, albeit within relatively simple models), analytic calculations, preliminary physics assessments and, finally, a more detailed engineering design study. At each stage, the design has been iterated to arrive at the solution we describe here, and that which is used for the detailed physics study described in the following section.

One of the fundamental differences between a tight aspect ratio tokamak power plant design and one of conventional aspect ratio is that there is no room to accommodate tritiumbreeding blankets on the inboard side of a ST. Fortunately, for sufficiently tight aspect ratios $(\lesssim 1.8)$, a tritium-breeding ratio greater than unity can nevertheless be achieved [7]. This is one of the main differences between tight and conventional
Table 1. Base-line parameters for the STPP design.

\begin{tabular}{ll}
\hline Parameter & Value \\
\hline Major radius/minor radius $(\mathrm{m})$ & $3.42 / 2.44$ \\
Elongation & 3.2 \\
Triangularity & 0.55 \\
Plasma current (MA) & 31 \\
Centre rod current $(\mathrm{MA})$ & 30.2 \\
Safety factor on axis, at edge & 3,15 \\
Line-average, central density $\left(\times 10^{19} \mathrm{~m}^{-3}\right)$ & $10.8,12.6$ \\
Greenwald density $\left(\times 10^{19} \mathrm{~m}^{-3}\right)$ & 16.6 \\
Average temperature $(\mathrm{keV})$ & 22 \\
$\beta$ (\%), $\beta_{\mathrm{N}}$ & $59,8.2$ \\
Internal inductance, $l_{\mathrm{i}}(2)$ & 0.21 \\
$Z_{\text {eff }}$ & 1.6 \\
Fusion power $(\mathrm{GW})$ & 3.1 \\
Current drive power $(\mathrm{MW})$ & $50-60$ \\
Auxiliary current drive $(\mathrm{MA})$ & 2.3 \\
Pressure driven current $(\mathrm{MA})$ & 28.7 \\
Confinement factor, $H_{\mathrm{IPB} 98(y, 2)}$ & 1.6 \\
Relative He confinement, $\tau_{\mathrm{He}}{ }^{*} / \tau_{E}$ & 4 \\
Average neutron wall loading $\left(\mathrm{MW} \mathrm{m}{ }^{-2}\right)$ & 3.5 \\
Peak neutron wall loading $\left(\mathrm{MW} \mathrm{m}^{-2}\right)$ & 4.6 \\
\hline
\end{tabular}

aspect ratio tokamaks, and is a defining feature of the STPP design. A point to note, therefore, is that one cannot evolve the design smoothly from a tight aspect ratio power plant design to a conventional tokamak design: there is a discontinuity in the design choice, which has significant implications as we discuss below.

Here, of course, we consider a ST design with no inboard breeding. Increasing the aspect ratio for a fixed plasma volume would increase the radial build of the device, making it more expensive. This points towards a desire to minimize the aspect ratio. On the other hand, reducing the aspect ratio reduces the size of the hole in the centre of the toroidal plasma. This hole must accommodate a current-carrying rod to provide the toroidal magnetic field, and we wish to maximize its radius in order to minimize the Ohmic dissipation in the rod (which has implications for both the cooling requirements of the rod and the economics through its impact on the re-circulating power fraction). As a result, there is a minimum aspect ratio of $A \sim 1.4$, below which the cost of electricity rises sharply. This is the value we choose for our study, which therefore complements a similar study by the ARIES team who considered $A=1.6$ [9-11]. It is interesting to note that both these choices are consistent with the results of systems codes analyses, which point towards a broad minimum in the cost of electricity in the region of aspect ratio $A=1.5$ (for normal-conducting toroidal field coils) [16].

The aspect ratio is one of the main parameters that guide the design; another is the fusion power. The majority of studies for power plants are performed for units that deliver $\sim 1 \mathrm{GW}$ of electrical power to the grid. To enable comparisons, we adopt this value. Studies of the power cycle conclude that the corresponding fusion power should be in the region of $3 \mathrm{GW}[8]$.

The remainder of the design parameters follows from these two choices. The size is determined by the average neutron wall loading, which is restricted to below $3.5 \mathrm{MW} \mathrm{m}^{-2}$. Furthermore, we shall see that it is desirable to maximize the plasma elongation, $\kappa$, in order to maximize the bootstrap current. A combination of the tight aspect ratio and hollow 
plasma current profile that we find for our solution, permits us to operate at $\kappa=3.2$. This is much higher than would be generally considered for a conventional aspect ratio device. Nevertheless, we shall see later that this value is almost naturally stable, with only modest requirements for vertical feedback control. The above discussion provides sufficient information to determine the major radius of the device, which is $R_{\mathrm{g}}=3.42 \mathrm{~m}$.

We now consider the bootstrap current, which we would like to maximize. An approximate scaling for the bootstrap current, $I_{\mathrm{bs}}$, is

$$
I_{\mathrm{bs}} \propto \beta_{\mathrm{N}} I_{\mathrm{rod}} h(\kappa),
$$

where $I_{\text {rod }}$ is the current in the centre rod and the normalized $\beta, \beta_{\mathrm{N}}=\beta(\%) a B_{\mathrm{g}} / I_{\mathrm{p}}$, (for the purposes of this definition, $\beta$ is measured as a percentage, the plasma minor radius, $a$, is in metres, the vacuum magnetic field at the geometric axis, $B_{\mathrm{g}}=\mu_{0} I_{\mathrm{rod}} / 2 \pi R_{\mathrm{g}}$ is in tesla and the plasma current, $I_{\mathrm{p}}$ is in MA). The function $h(\kappa)$ increases monotonically with elongation; its precise form depends on the current profile, but it is typically close to linear. Operating with $\beta_{\mathrm{N}}$ close to the ideal MHD stability limit (i.e. $\beta_{\mathrm{N}}=8.2$ [17]) we find that we require $I_{\text {rod }} / I_{\mathrm{p}}$ to be close to 1 to achieve $90 \%$ pressure-driven current. To determine the absolute values of these currents, we take the total stored energy in the plasma to be that required to provide the necessary fusion power. From the value of $\beta_{\mathrm{N}}$, we then find that the currents are required to be $\sim 30-31$ MA. We choose to operate at a relatively low line-averaged density of $1.08 \times 10^{20} \mathrm{~m}^{-3}$ ( $65 \%$ of the Greenwald limit), so that we are able to drive the remaining $10 \%$ of the plasma current with an acceptable level of auxiliary power. This level of external current drive also allows a degree of current profile control to help optimize the plasma stability.

One measure of the overall consistency is to compare the required confinement time with the predictions from the various scaling laws. We find, for example, that a confinement time $\sim 1.6$ times the prediction of the IPB98 $(y, 2)$ scaling law [18] is required. This could be reduced in principle by raising either the plasma current or the plasma density, but this would increase the requirements from the current drive system.

\section{Physics studies}

In the previous section we described the processes which led to our base-line design. In this section we describe those plasma physics issues that we have looked at in more detail in order to: (a) put the design on a firmer theoretical basis and (b) identify those areas which require further work in the future. Thus, we start in section 3.1 with a calculation of the equilibrium and its vertical stability properties. Then, in section 3.2 we explore the MHD stability, concentrating mainly on ideal MHD, but also making some remarks regarding neoclassical tearing modes. We shall find that MHD stability places constraints on the current density profile, and in section 3.3 we describe possible schemes for driving the desired current, and make predictions for the current drive power that would be required. In section 3.4 we consider issues related to the plasma confinement, including the $\alpha$-particle confinement, and perform one-dimensional transport modelling of a discharge. We also explore the linear micro-stability of the plasma using a gyro-kinetic code (GS2) [19], taking account of the finite $\beta$, to

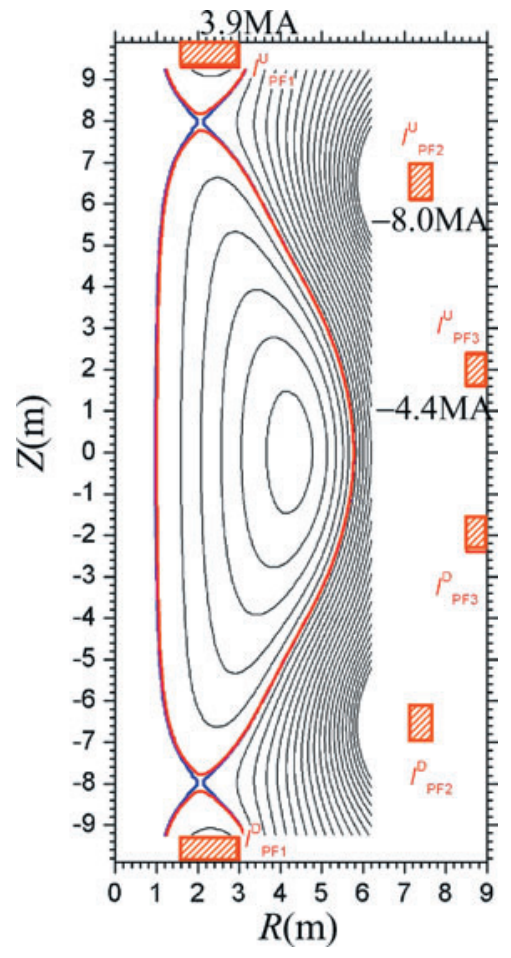

Figure 1. Free boundary equilibrium reconstruction for the STPP design. The hatched squares show the cross-sections of the poloidal field coils, and their associated currents are indicated alongside.

identify which modes may be responsible for driving turbulent transport. We consider issues related to fuelling in section 3.5. Finally, in section 3.6 we address the plasma exhaust and make predictions for the heat loads that a divertor system would need to handle.

\subsection{Equilibrium properties}

In order to minimize the heat loads to divertor target plates, we choose to operate in an up-down symmetric double null configuration, the equilibrium for which is shown in figure 1 . Three pairs of poloidal field coils are used to produce the desired plasma shape, the key features of which are the tight aspect ratio and high elongation. Such a high elongation would be extremely difficult to control in a conventional aspect ratio tokamak, but one of the features of a ST is that it has a high natural elongation and high values are achievable with no need for vertical feedback control. In addition, the pressure-driven current tends to provide a hollow current profile, and this is also favourable for high elongation. To quantify the vertical stability, we therefore need to address the pressure and current profiles used to construct the equilibrium shown in figure 1.

The electron temperature and density profiles are shown in figure 2 (for simplicity we assume that the ion and electron temperatures are equal, and this is supported by the onedimensional transport modelling that we describe later). We have adopted a rather flat density profile, typical of those observed in MAST H-modes, for example, and a somewhat more peaked temperature profile. The pressure-driven current profile is calculated for the specified pressure profile using the SCENE code. This is a fixed boundary equilibrium code that is designed to calculate equilibria with a self-consistent 

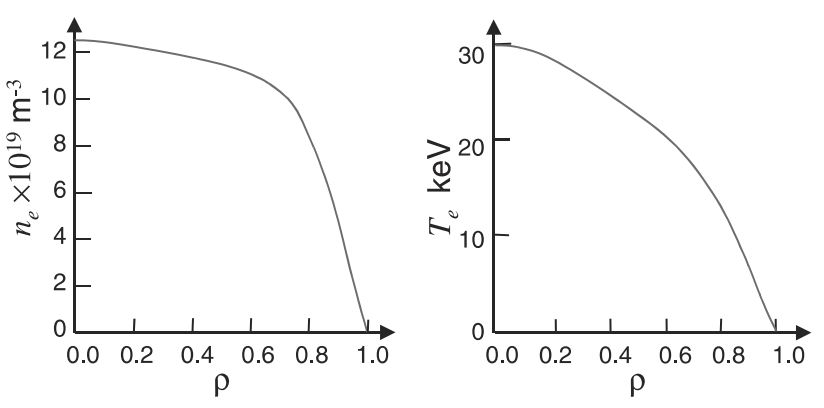

Figure 2. The assumed electron density, $n_{\mathrm{e}}$, and temperature $T_{\mathrm{e}}$ profiles for the STPP equilibrium.

current profile, determined by the sum of the pressure-gradientdriven current densities and any specified externally driven current. The pressure-driven current fraction is calculated to be $92 \%$, of which $82 \%$ is due to the bootstrap current. The remainder is provided by the toroidal component of the diamagnetic current, which can be significant in a ST (its toroidal component is typically negligible in a conventional tokamak). Figure 3(a) shows the bootstrap current profile; figure $3(b)$ shows the total current density profile (required for MHD stability, as discussed in the next subsection); figure $3(c)$ shows the necessary auxiliary current drive profile and figure $3(d)$ shows the profile of the safety factor, $q$. Figure 3 illustrates an extremely important feature of the ST: even though the current profile is hollow, the $q$-profile remains monotonic. This is largely because the magnetic shear due to the magnetic geometry is very strong at the plasma edge, and therefore a large current density can be tolerated there without the shear becoming negative. The internal inductance for this equilibrium, defined by either

$$
l_{\mathrm{i}}^{(2)}=\frac{L^{2}}{2 \pi \mu_{0}^{2} I_{\mathrm{p}}^{2} V} \oint B_{\theta}^{2} \mathrm{~d} V
$$

or

$$
l_{\mathrm{i}}^{(3)}=\frac{4 \pi}{\mu_{0}^{2} I_{\mathrm{p}}^{2} R_{\mathrm{g}}} \oint B_{\theta}^{2} \mathrm{~d} V,
$$

is calculated to be $l_{\mathrm{i}}^{(2)}=0.21$ or $l_{\mathrm{i}}^{(3)}=0.12$. Here, we have defined the poloidal circumference of the last closed flux surface, $L$, the plasma volume, $V$, and the poloidal field, $B_{\theta}$. The calculated current profile from SCENE was used as the input to the free boundary equilibrium code used to calculate the equilibrium shown in figure 1.

From the free boundary equilibrium we can assess the vertical stability of the equilibrium, for which we use the PACE filament code [20]. This code models the plasma as a bundle of individual current elements linked inductively with the surrounding conductors. We take into account the position of the vessel wall (which we shall discuss in the following subsection) and its effective resistivity. The equilibrium is then found to be extremely close to marginal stability, even in the absence of any feedback control. Nevertheless, it is just unstable, with a predicted growth time of $10 \mathrm{~ms}$, but the requirements of any feedback control system to maintain this equilibrium are modest. This growth time can be quantified by calculating the vertical stability index, $f_{\mathrm{s}}[21]$, which is the ratio of stabilizing to destabilizing vertical force gradients; we find $f_{\mathrm{s}}=3.5$.

\subsection{MHD stability}

We first discuss the high toroidal mode number, $n$, ballooning stability, which is illustrated by the $s-\alpha$ plots at three different flux surfaces, shown in figure 4 . The magnetic shear, $s$, and normalized pressure gradient, $\alpha$, are given by

$$
s=\left(R_{2}-R_{0}\right) \frac{R_{0} B_{\theta}}{q} \frac{\mathrm{d} q}{\mathrm{~d} \psi}, \quad \alpha=-2 \mu_{0} \frac{\left(R_{2}-R_{0}\right)^{2}}{B_{\theta}} \frac{\mathrm{d} p}{\mathrm{~d} \psi},
$$

where the poloidal field, $B_{\theta}$, and the major radius, $R_{2}$, are measured at the outboard mid-plane of the flux surface; $R_{0}$ is the major radius of the magnetic axis, $p$ is the plasma pressure and $\psi$ is the poloidal flux per radian. The figures show the $s$ and $\alpha$ values for the power plant equilibrium marked by a star. The curves provide a measure of the marginal stability boundary, obtained by scaling $s$ and $\alpha$ from the equilibrium values ${ }^{1}$. The three flux surfaces chosen are representative of the edge (high magnetic shear, $\psi_{\mathrm{N}}=0.98$ ) region, the confinement region (around the mid-radius, $\psi_{\mathrm{N}}=0.5$ ) and close to the magnetic axis $\left(\psi_{\mathrm{N}}=0.05\right)$. Note that the plasma has access to the so-called second stability region at high $\alpha$ across the full radius. This is achieved because of the hollow current profile, and indeed the requirement of ballooning stability provides the main constraint on the current profile. Thus, by taking current away from the core and placing it towards the plasma edge we (a) raise the central $q$, thus stabilizing the core to ballooning and Mercier modes [22] and (b) lower the edge shear so that the equilibrium can squeeze under the 'nose' of the $s-\alpha$ stability diagram and provide access to the second stable region for ballooning modes.

We next address the stability to the low $n$ kink modes, considering the stability to $n=1,2$ and 3 [23]. We find that kink stability is rather sensitive to the central safety factor, $q_{0}$, and that if $q_{0}<q_{\mathrm{c}}$, where $q_{\mathrm{c}} \approx 3$, there is a strong internal kink mode. Figure 5 shows how this is stabilized by having an infinitely conducting wall on the plasma as $q_{0}$ is increased above 3 . Thus, by ensuring that the conducting wall of the vacuum vessel lies sufficiently close to the plasma, we find that the low $n$ modes can also be stabilized. For our example equilibrium, this requires a conformal wall at a distance $\sim 15 \%$ of the minor radius from the plasma surface, and this can be accommodated in the design. There remains an issue with the resistive wall mode of course, and this is a subject of ongoing research. We make no quantitative predictions here, but evidence from conventional tokamaks does suggest that these modes can be controlled in principle by a suitable feedback system.

Neoclassical tearing modes (NTMs) often provide the effective pressure limit in both conventional tokomaks and STs $[24,25]$. It is, therefore, natural to ask what limitations they are likely to cause in any high $\beta$ power plant design. Unfortunately, the models for the onset of NTMs are not sufficiently developed to allow accurate, quantitative predictions for future machines, but we can make some estimates as follows. Neglecting threshold effects, we can model the evolution of a magnetic island of width $w$ according to the equation:

$$
\frac{\tau_{r}}{r^{2}} \frac{\mathrm{d} w}{\mathrm{~d} t}=\Delta^{\prime}+\frac{D_{\mathrm{bs}}}{w}+\frac{D_{\mathrm{G}}}{w},
$$

1 The ballooning parameter, $\theta_{0}$, has been set to the out-board midplane poloidal angle in these figures for simplicity. Nevertheless, a scan over this parameter shows that the equilibrium is stable to all $\theta_{0}$ values. 

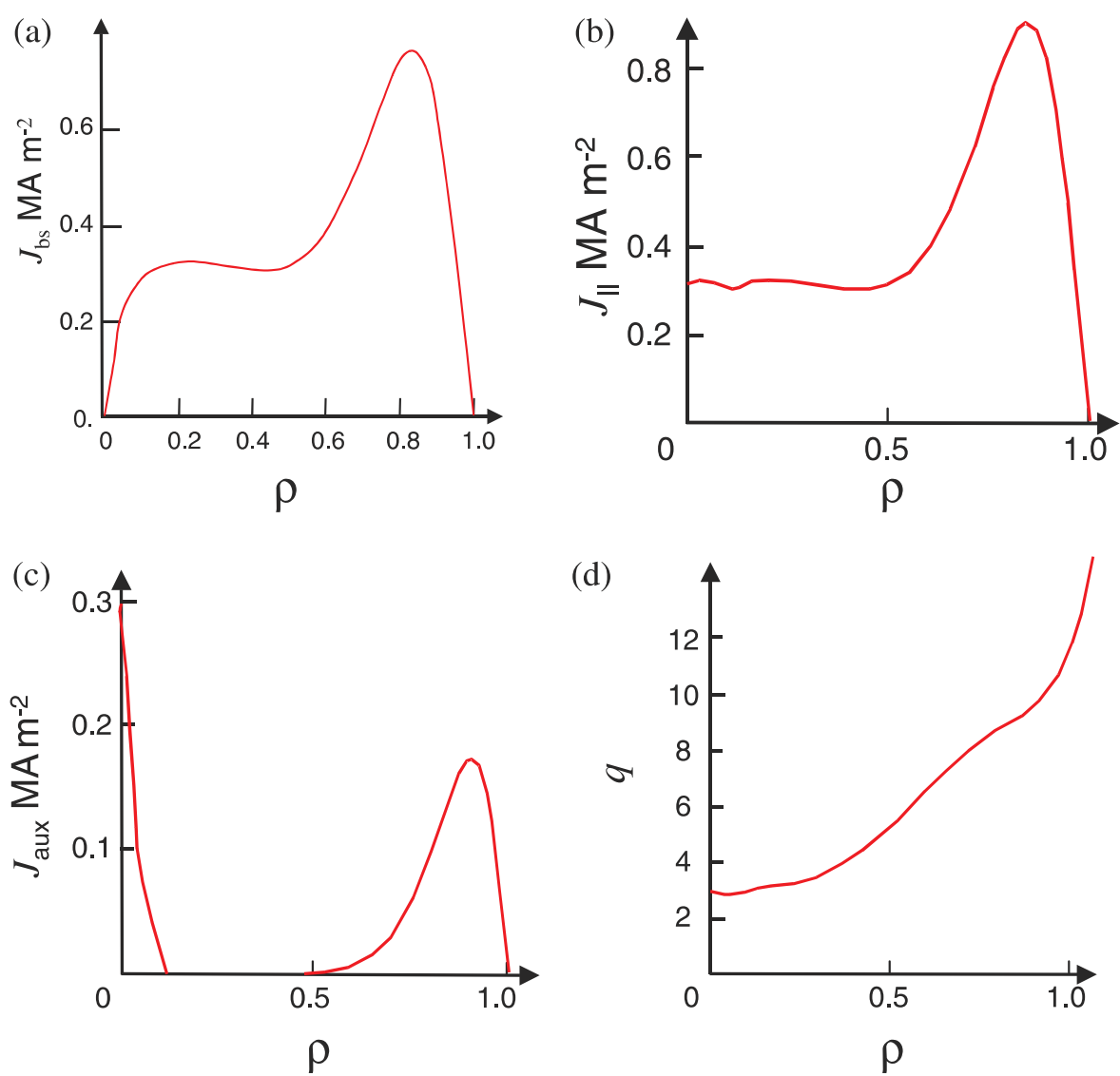

(d)

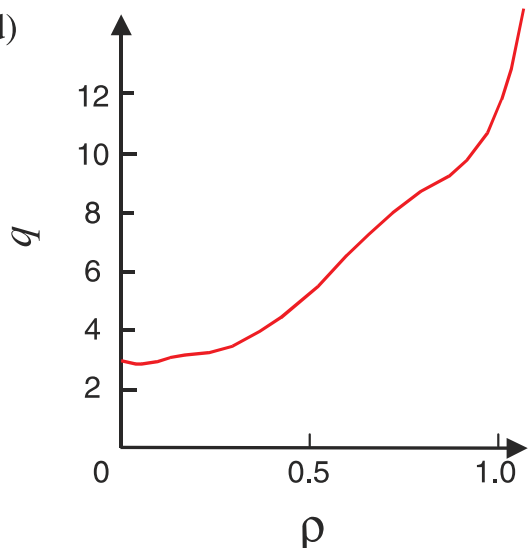

Figure 3. Radial profiles of $(a)$ the bootstrap current density, $(b)$ the total current density, $(c)$ the required auxiliary current drive and $(d)$ the safety factor.

where $r$ is the radius of the rational surface associated with the NTM and $\Delta^{\prime}$ is the standard parameter, familiar from the classical theory of tearing modes, which measures the free energy in the current profile to drive the tearing mode. For a neoclassical tearing mode, $\Delta^{\prime}<0$ and $D_{\mathrm{bs}}>0$, where $D_{\text {bs }}$ measures the drive from the bootstrap current perturbation $[25,26]:$

$$
D_{\mathrm{bs}}=3.2 \frac{\left\langle\boldsymbol{J}_{\mathrm{bs}} \cdot \boldsymbol{B}\right\rangle q}{q^{\prime} f p} \beta_{\theta} .
$$

Here, $\boldsymbol{J}_{\mathrm{bs}}$ is the bootstrap current, $\boldsymbol{B}$ the magnetic field, $q$ the safety factor, $p$ the plasma pressure and $f=R B_{\phi}$, where $R$ is the major radius and $B_{\phi}$ is the toroidal component of the magnetic field. A prime denotes a derivative with respect to poloidal flux. The Glasser term, proportional to $D_{\mathrm{G}}$, is negative, but is typically much smaller than the bootstrap term for conventional aspect ratios [25-27]:

$$
D_{\mathrm{G}}=\frac{6 D_{\mathrm{R}}}{\alpha_{\mathrm{s}}-H},
$$

where $\alpha_{\mathrm{s}}=0.5+\left(0.25-D_{\mathrm{M}}\right)^{1 / 2}$ and $D_{\mathrm{R}}$ and $D_{\mathrm{M}}=$ $D_{\mathrm{R}}+H(1-H)$ are the resistive and ideal interchange mode parameters, respectively. However, $D_{\mathrm{G}}$ increases with aspect ratio and plasma shaping, so there is the possibility at tight aspect ratio that the Glasser term can exceed the bootstrap term. The NTM is then unconditionally stable. In figure 6 we show the results of calculations for each of these terms as a function of the safety factor, $q$, which serves as a radial variable. If we first take a conventional tokamak equilibrium with aspect ratio $A=3$ and safety factor on-axis $q_{0}=1$, we find that the bootstrap term exceeds the Glasser term and NTMs are a possibility (see figure 6(a)). However, if we now do the same calculation for the STPP equilibrium (shown in figure $6(b)$ ), we find that the Glasser term exceeds the bootstrap term, suggesting that NTMs are unconditionally stable. One should be wary here as there are significant uncertainties in the values of the coefficients of $D_{\mathrm{bs}}$ and $D_{\mathrm{G}}$, although the values used here have been benchmarked against MAST data [26]. There is, however, another point in favour of the STPP equilibrium: the most dangerous NTMs are associated with low order rational surfaces, and these have been eliminated from the ST equilibrium by maintaining $q>3$ (see figure 3 ).

\subsection{Current drive}

Approximately $8 \%$ of the plasma current (2.3 MA) remains to be driven by an auxiliary current drive scheme, and we have considered providing this by both NBI and radiofrequency waves. The required current drive profile is shown in figure $3(c)$, where it can be seen that two contributions are needed: approximately $140 \mathrm{kA}$ near the magnetic axis and 2.2 $\mathrm{MA}$ in the outer region of the plasma.

We first consider the NBI. A suitable system has been designed based on detailed modelling with the full orbit code, LOCUST (Larmor Orbit Code for Use in Spherical Tokamaks) [28]. This is a Monte Carlo fast ion gyro-orbit code, which 

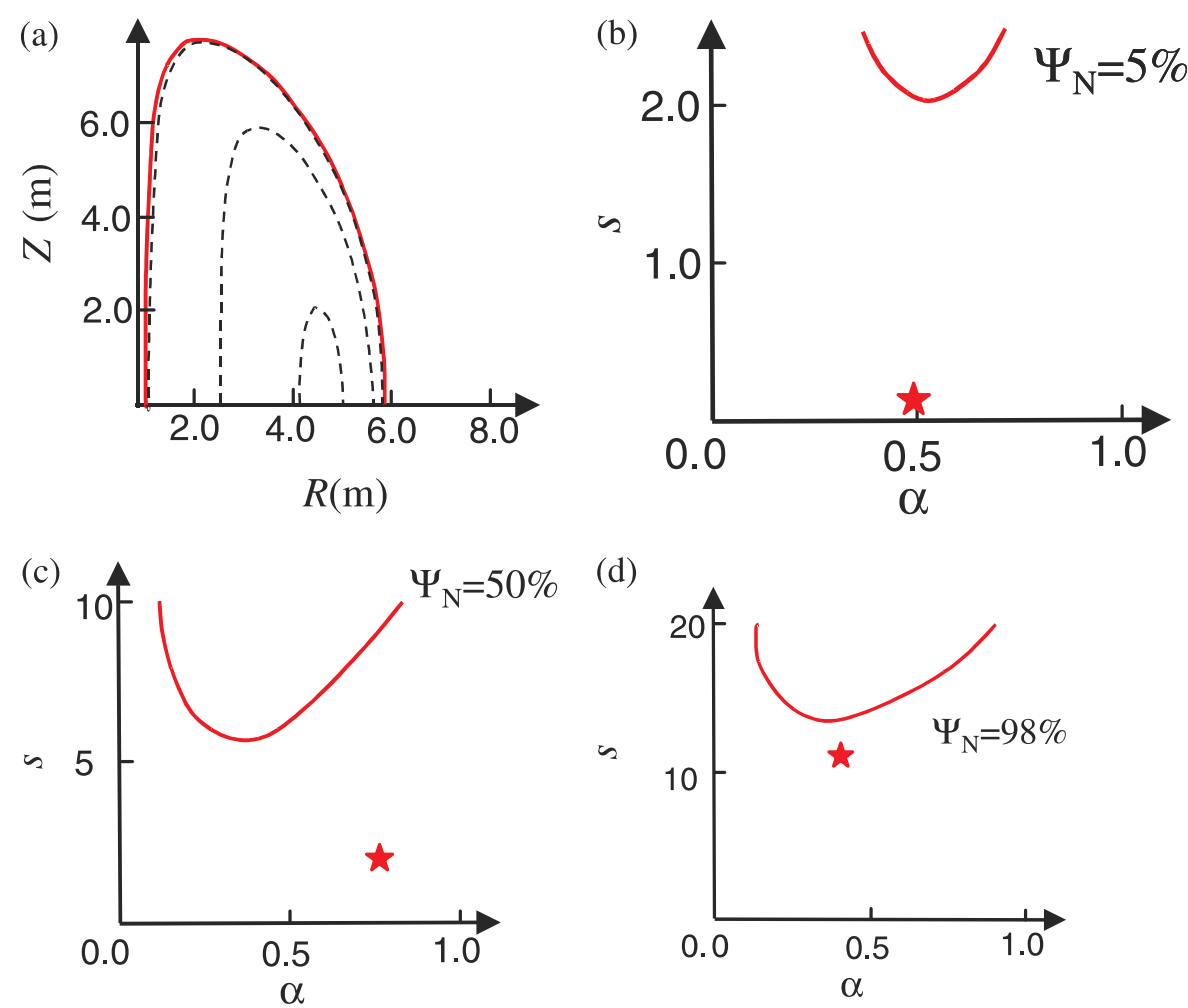

Figure 4. Ballooning stability analyses. (a) shows the positions of the flux surfaces (- - - ) relative to the plasma boundary ( - ), which has been approximated by a limiter geometry for the fixed boundary equilibrium solver used for the stability study. $(b)-(d)$ show the position of the equilibrium for each of the three flux surfaces $\left(\psi_{\mathrm{N}}\right.$ is the poloidal flux normalized to its edge value) in the $s-\alpha$ stability diagram $(\star)$ relative to the marginal stability boundary (unstable above the curve).

(a)

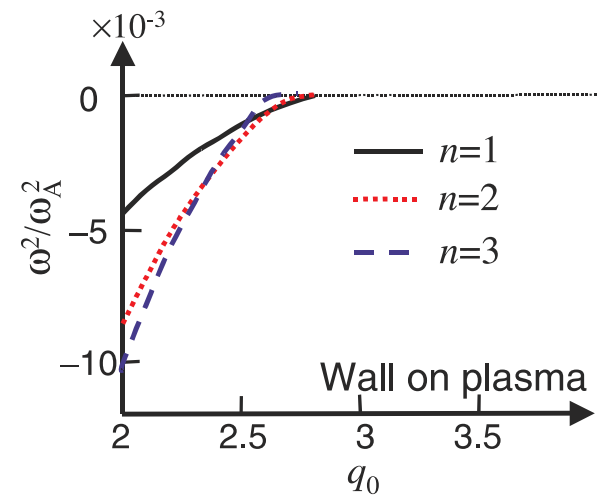

(b)

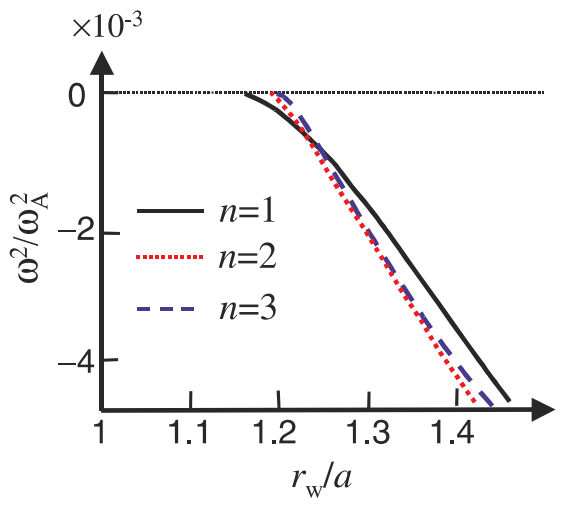

Figure 5. Stability for $n=1,2$ and 3 modes $(a)$ with an infinitely conducting wall on the plasma as a function of the central safety factor, $q_{0}$ and $(b)$ fixing $q_{0}=2.9$ and varying the wall position.

calculates the collisional evolution of the fast ion distribution resulting from NBI including electron drag, scattering with the bulk and impurity ions and charge-exchange-driven cross field transport and loss. The code has been benchmarked both against MAST data [15], and with the extended guiding centre NBI model in TRANSP [29]. The result of the calculations for STPP is that the current can be provided with two beam systems, as follows. The current drive on-axis can be achieved with a $20 \mathrm{MW}, 0.5 \mathrm{MeV}$ negative ion beam, injected at a tangency radius, $R=4.0 \mathrm{~m}$, which is just inside the magnetic axis. Much of this beam is stopped before it reaches this tangency radius, so its current drive efficiency is relatively low. The current drive in the outer region can be provided by $40 \mathrm{MW}$ of an $80 \mathrm{keV}$ system at a tangency radius $R=5.65 \mathrm{~m}$. For this case, we find that trapping effects are minimized and the current drive efficiency is maximized if the beam is aligned with the magnetic field lines in the region where the current drive is required. This leads us to position this outer beam at an angle of $60^{\circ}$ to the horizontal. Figure 7 shows that a good match to the MHD stable current profile (i.e. that used for the MHD stability studies of the previous section) is obtained when the calculated neutral beam current drive is added to the pressure driven current.

Although neutral beams can provide the required current drive, there are disadvantages, in that large ports would be required and also related, large equipment would need to 
(a)

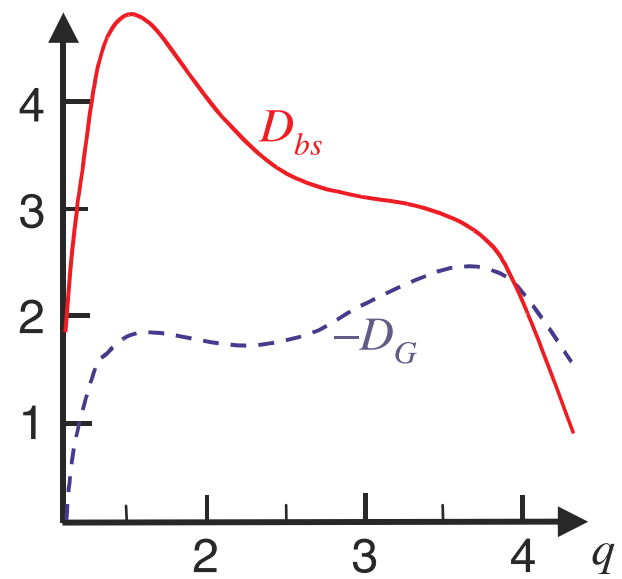

(b)

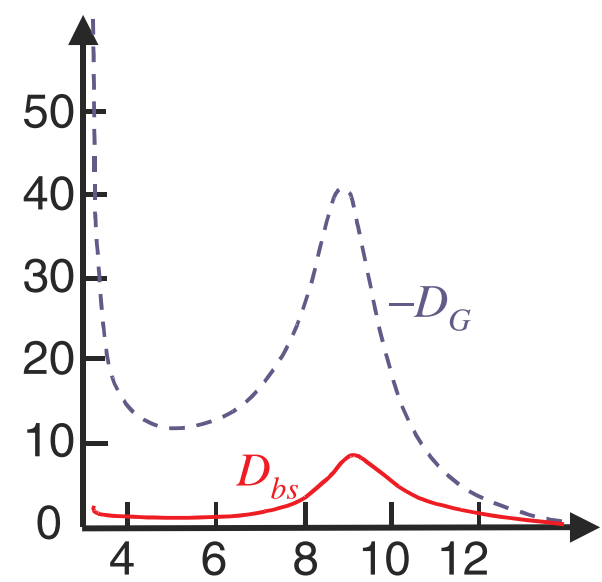

Figure 6. Comparison of the bootstrap current drive (_- term with the Glasser term [27] (- - - -) in the neoclassical tearing mode evolution equation for $(a)$ a conventional, aspect ratio $=3$ equilibrium and $(b)$ the STPP equilibrium.

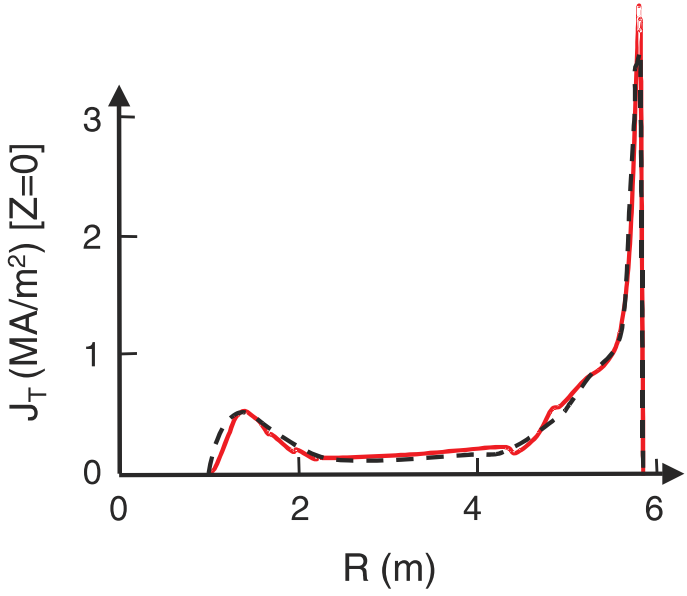

Figure 7. MHD stable current profile (- - -) compared to the calculated current profile from the sum of NBI and pressure-driven currents, plotted across the midplane.

be placed close to the tokamak, where space is limited. Calculations show that it is possible to accommodate the neutral beam system, in principle, and a tritium breeding ratio of $\sim 1.1$ can be achieved even allowing for the ports [7]. Nevertheless, it may be beneficial to employ radio-frequency waves to complement at least part of the neutral beam system. Electron cyclotron frequency waves are particularly attractive as these can propagate through a vacuum so that there is no need to position an antenna close to the plasma surface, and they do not require a large port. In addition, the gyrotrons for generating the waves can be located far from the tokamak. We have considered two systems: direct use of conventional electron cyclotron waves and also electron Bernstein waves.

Let us first consider conventional electron cyclotron waves. A feature specific to the ST is that on the outboard midplane, the toroidal and poloidal components of the magnetic field can become comparable, particularly at high $\beta$. Thus, while in conventional tokamaks the total magnetic field decreases monotonically with increasing major radius, the magnetic field in our STPP equilibrium has a minimum close to the magnetic axis (see figure 8 ). This provides one

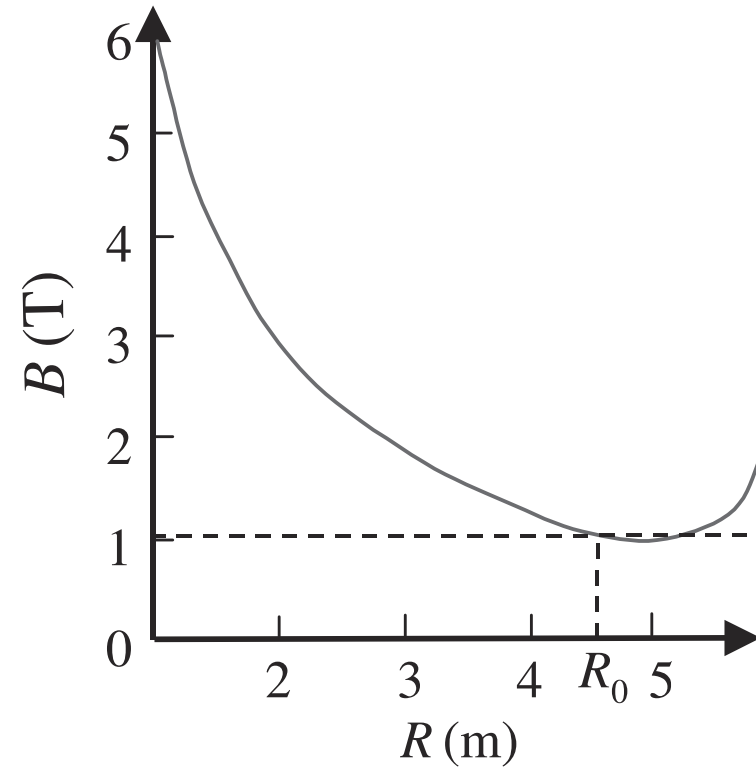

Figure 8. Total magnetic field as a function of the plasma major radius across the tokamak midplane; $R_{0}$ indicates the position of the magnetic axis.

problem for driving current near the magnetic axis, as lower harmonics of the waves can be resonant with the magnetic field at a larger major radius, leading to premature absorption before the magnetic axis is reached. An additional problem associated with the propagation of electron cyclotron waves in STs, in general, is that the magnetic field is relatively low compared with the plasma density. This means that it is often difficult, or even impossible, to arrange for the waves to exceed the cut-off frequency (i.e. where the wave frequency, which is the electron cyclotron frequency or a low harmonic of it, exceeds the plasma frequency). Nevertheless, a possible scheme has been identified for the current drive on-axis, employing electron cyclotron frequency waves at $130 \mathrm{GHz}$ (4th harmonic damping near the magnetic axis). The required power was evaluated using the BANDIT-3D ray tracing/Fokker Planck code [30]. The result is that $15 \mathrm{MW}$ would be required to drive the $140 \mathrm{kA}$ of on-axis current, which is comparable with the 


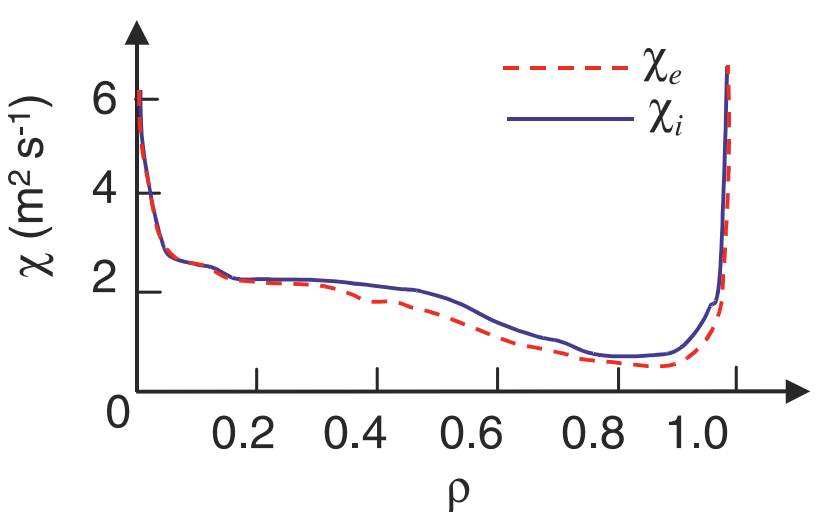

Figure 9. Thermal diffusivities required to produce the temperature profiles assumed for the electrons and ions.

neutral beam requirements for this component. Turning to the current required towards the plasma edge, electron cyclotron waves deposit a significant fraction of their momentum into the perpendicular velocity component of the electrons. Therefore, particle trapping effects tend to reduce the resulting current drive efficiency. Indeed, we find that electron cyclotron waves are not a suitable option for the edge current drive for precisely this reason.

Electron Bernstein waves (EBW) do not travel through a vacuum so, to avoid placing an antenna close to the plasma surface, we exploit the $\mathrm{O}-\mathrm{X}-\mathrm{B}$ conversion process $(\mathrm{O}$-mode to $\mathrm{X}$-mode to Bernstein mode). This is expected to be very efficient near the edge of the plasma [31], and will be tested on MAST. The resulting Bernstein wave could then propagate through the plasma until it encounters its (Doppler-shifted) resonance. There are two reasons why we are particularly interested in EBW: they do not have a low density cut-off and they tend to damp more on those electrons with a larger component of the velocity parallel to the magnetic field. The latter property would be expected to reduce the effects of trapping, and so provide more efficient current drive in the outer region of the plasma. Preliminary calculations have been performed which derive the damping using a relativistic dispersion relation and then employ an extension of the Fisch-Boozer method to estimate the current drive [32,33]. Assuming trapping effects are unimportant here (they are included in the NBI and ECCD predictions), relatively high current drive efficiencies in the range $0.1-0.15 \mathrm{AW}^{-1}$ are predicted at the edge, but these predictions should be compared with Fokker-Planck calculations, including trapping effects in the future (in particular the Okhawa contribution to the current [34]). Strong off-axis absorption causes problems with using EBW for core current drive, and we have not yet identified a viable scenario for this.

\subsection{Confinement}

We now discuss issues related to the plasma confinement. As we stated earlier, the design is based upon a prediction of the global confinement time, which requires a relatively modest enhancement of a factor 1.6 above the $\operatorname{IPB} 98(y, 2)$ scaling law [18]. This scaling law has been derived largely from conventional tokamaks, but nevertheless provides a reasonable description of MAST data [15]. H-mode access appears to be possible, if it is required: the latest scaling law [35] predicts a threshold power of $50 \mathrm{MW}$. In this section we go beyond scaling laws, and report some initial studies of the details of the transport processes which will ultimately determine the confinement time.

We chose the density and temperature profiles shown in figure 2 in order to satisfy the ideal MHD stability constraints. In practice, these profiles would be determined by a combination of the sources (particles or heat) and the heat/particle diffusivities but, in the absence of a reliable, quantitative model, these are uncertain. Instead, we deduce the thermal diffusivities, which will be required to provide the temperature profile we assumed (figure 2), and check that they are sensible. This has been done using the ASTRA transport code [36], which employs the self-consistent heating from the fusion-produced $\alpha$-particles. It is found that the heating is approximately evenly distributed between electrons and ions. The thermal diffusivities (shown in figure 9) are a little below, but roughly consistent with, crude mixing length estimates of the turbulent transport (see later). Moreover, they are similar to experimental values found for the MAST H-mode discharge \#6953 [15], although the plasma parameters of this pulse are quite different from those of STPP.

We have also performed a time-dependent transport analysis for the STPP using the ASTRA code. In this study, we started from an initial low density, low plasma current discharge and then ramped these up to their operating values for the required fusion power $(\sim 3 \mathrm{GW})$. ASTRA then self-consistently solves for the evolution of the temperature profiles, fusion power, helium ash accumulation and bootstrap current. The thermal diffusivity model we adopt consists of a contribution from the Canonical Profiles Transport model [37] to which we add a constant contribution, $\chi_{0}$. This constant is adjusted so that the confinement time is the required factor of 1.6 above the prediction of the IPB98 $(y, 2)$ scaling law (the pure Canonical Profiles model has little power degradation and so would result in large enhancement factors for the power plant). The evolution of some of the more important parameters is shown in figure 10, where it can be seen that the equilibrium does settle down to a steady state with a fusion power just below $3 \mathrm{GW}$ and a pressure-driven current fraction of $90 \%$. The modelling predicts that the electron and ion temperatures are approximately the same, justifying our earlier assumption. A sensitivity study, comparing different transport models, has not been performed. However, we expect that there will be a strong dependence of the fusion power on the confinement enhancement factor, but the dependence on the radial profile of the thermal diffusivity is expected to be much weaker.

We now consider the types of micro-instabilities that may be responsible for the transport in STPP. We choose to analyse a flux surface in the confinement region, at about half the minor radius (figure $11(a)$ ). Figure 11(b) shows the strong variation of the magnetic field around this surface.

Linear micro-stability has been studied using the electromagnetic gyro-kinetic initial value code GS2 [19]. If we consider purely electrostatic modes, and switch off electromagnetic effects in the code, no instabilities are found. However, when electromagnetic effects are retained, GS2 does predict the presence of tearing parity micro-instabilities. Similar tearing parity modes have been identified in previous 


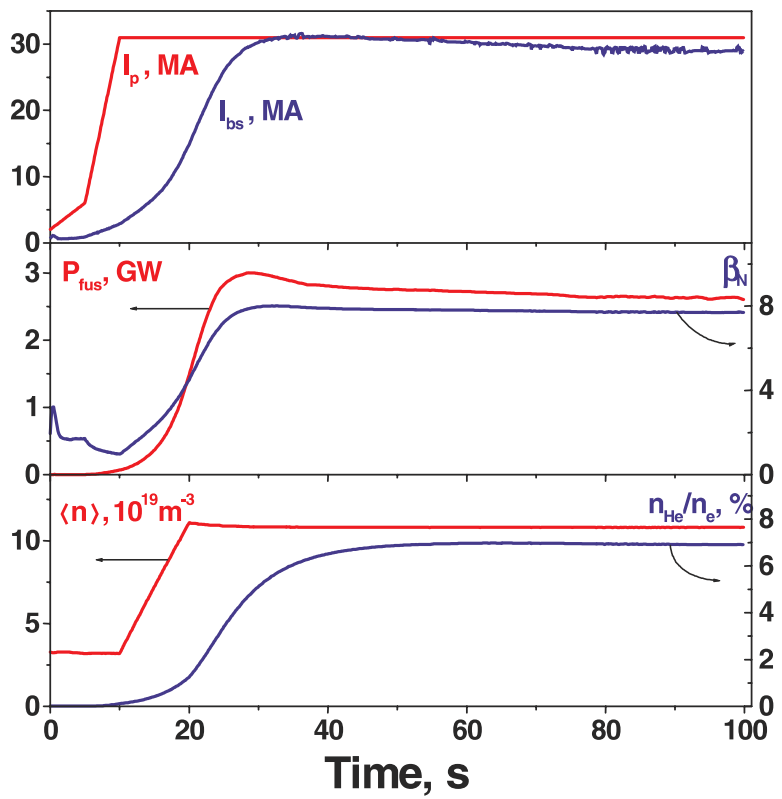

Figure 10. Time dependence of plasma current $\left(I_{\mathrm{p}}\right.$, imposed $)$, bootstrap current $\left(I_{\mathrm{bs}}\right.$, calculated), fusion power ( $P_{\text {fus }}$, calculated), $\beta_{\mathrm{N}}$ (calculated), average density ( $\langle n\rangle$, imposed) and He density fraction $\left(n_{\mathrm{He}} / n_{\mathrm{e}}\right.$, calculated $)$. (a)

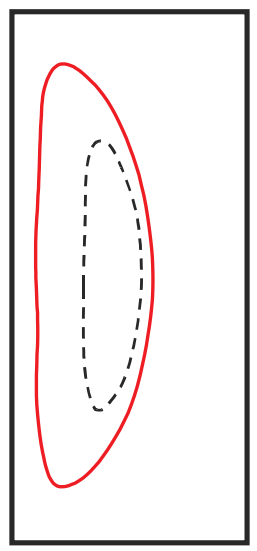

(b)

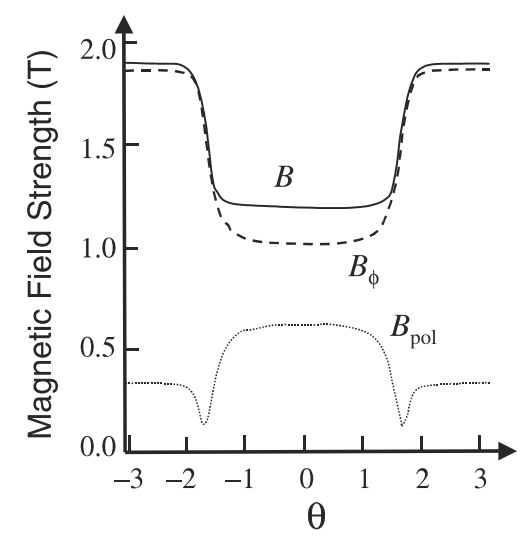

Figure 11. (a) The STPP equilibrium surface where micro-stability has been studied. (b) Magnetic field strengths as a function of poloidal angle around this surface.

theoretical studies of high $\beta$, low aspect ratio tokamak equilibria [38].

The growth rates of the dominant modes are presented in figure $12(a)$ as a function of $k_{y} \rho_{\mathrm{i}}$ where $k_{y}$ is the wave-number in the direction which is perpendicular to the magnetic field and in the flux surface, and $\rho_{\mathrm{i}}$ is the ion Larmor radius on the flux surface, evaluated using the vacuum magnetic field strength on the magnetic axis. When $k_{y} \rho_{\mathrm{i}}<1.0$ (the ion temperature gradient instability, or ITG, regime), both the electron and ion distribution functions are evolved using GS2. The resulting growth rates are indicated by open circles in figure 12. In this regime, the modes are found to be extremely extended along the field line $(\sim 100$ turns around the poloidal circumference). In the opposite regime, where $k_{y} \rho_{\mathrm{i}}>1.0$, we approximate the ion response as adiabatic and only solve for the electron distribution function. In this regime, relevant for electron temperature gradient (ETG) modes, the eigenmodes are much less extended along the field line. The growth rates are given by the full circles in figure 12. Finally, the closed square symbols indicate calculations where no instability was found. We note that the ballooning parameter $\theta_{0}$ (which characterizes the radial wave-number) was set to zero for these calculations (corresponding to the outboard midplane), but a scan at $k_{y} \rho_{\mathrm{i}}=0.4$ over the range $0<\theta_{0}<\pi / 2$ revealed only a weak dependence of the growth rate on this parameter.

It is interesting to compare the growth rates with the $E \times B$ shearing rate, $\omega_{\text {se }}$ [39]. For flute-like turbulence the shearing rate is given by

$$
\omega_{\mathrm{se}}=\frac{\Delta \Psi}{\Delta \phi} \frac{\partial^{2} \Phi}{\partial \Psi^{2}},
$$

where $\Phi$ is the electric scalar potential, $\Psi$ the poloidal flux, $\phi$ the toroidal angle, and the quantities preceded by $\Delta$ indicate the turbulence correlation length in that direction. Taking $\Delta \Psi=R B_{\theta} \Delta r$ and $\Delta \phi=\left(r B_{\phi} / R B_{\theta}\right) \Delta \theta$ gives

$$
\omega_{\mathrm{se}}=\frac{R^{2} B_{\theta}^{2}}{B_{\phi}} \frac{\Delta r}{r \Delta \theta} \frac{\partial^{2} \Phi}{\partial \Psi^{2}},
$$

where $r$ is the minor radius. If the turbulence is assumed to be isotropic at the location of interest (i.e. $\Delta r=r \Delta \theta$ ), the shearing rate depends strongly on the poloidal location chosen and is maximum on the outboard side of the flux surface (see figure 12). It is unclear how flow shear would be expected to influence these tearing parity, electromagnetic modes. Nevertheless, it is interesting to note that the shearing rate is at a level where it might be expected to affect the stability properties of the ITG modes, for which $k_{y} \rho_{\mathrm{i}}<1.0$ (i.e. where the shearing rate exceeds the linear growth rate).

Although mixing length estimates of the transport resulting from turbulence can often be misleading, they provide an indication of the level of transport that might be expected. If we ignore flow shear stabilization, such an estimate would predict a thermal diffusivity in the region $\sim 4 \mathrm{~m}^{2} \mathrm{~s}^{-1}$. Given the uncertainties, this is broadly within the range of what is required (see figure 9). Computationally challenging nonlinear calculations that include the effects of flow shear are required to make more rigorous estimates, but these have not yet been attempted.

Since most of the heating comes from the $\alpha$-particles, it is important to ensure that they are confined within the plasma for a significant fraction of the slowing down time. To explore this we have computed $\alpha$-particle losses using a full orbit code CUEBIT [40], taking account of a $\sim 1 \%$ ripple in the toroidal component of the magnetic field calculated from the engineering design. Collisional friction is included in the calculation. For the confinement of $\alpha$-particles, the nonmonotonic magnetic field (see figure 8 ) is a benefit, as it leads to a pinching of trapped particle orbits. Thus, although the drift orbit widths of $\alpha$-particles born near the magnetic axis can be a significant fraction of the minor radius, the effect of the non-monotonic magnetic field is to pinch the orbits as they cross the outboard midplane; such an orbit is shown in figure $13(a)$. For a particle born very close to the edge (as in figure $13(b)$ ), the guiding centre orbit width is negligible, and excursions from the birth flux surface are limited to a single Larmor radius. This results in a high degree of confinement. 

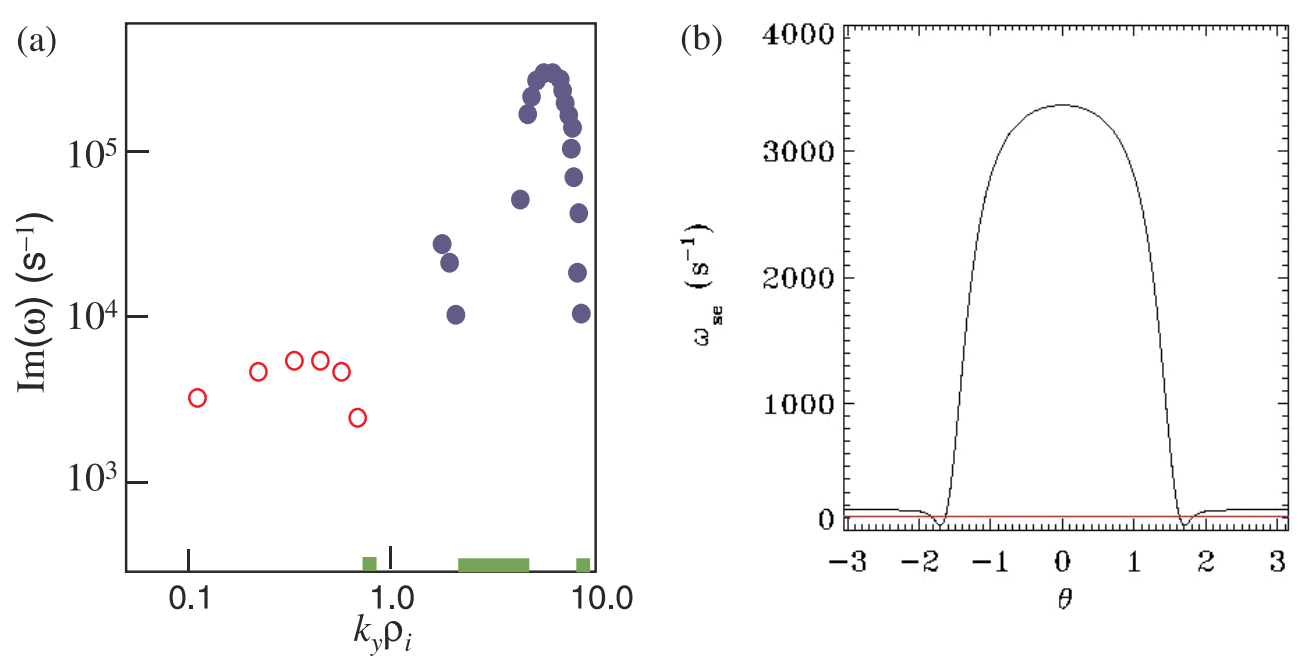

Figure 12. (a) Growth rates of tearing parity modes as a function of the normalized wavenumber $K_{y} \rho_{\mathrm{i}}$. $(b)$ Equilibrium $E \times B$ shearing rate on this surface (ignoring rotation) as a function of poloidal angle $\theta$.
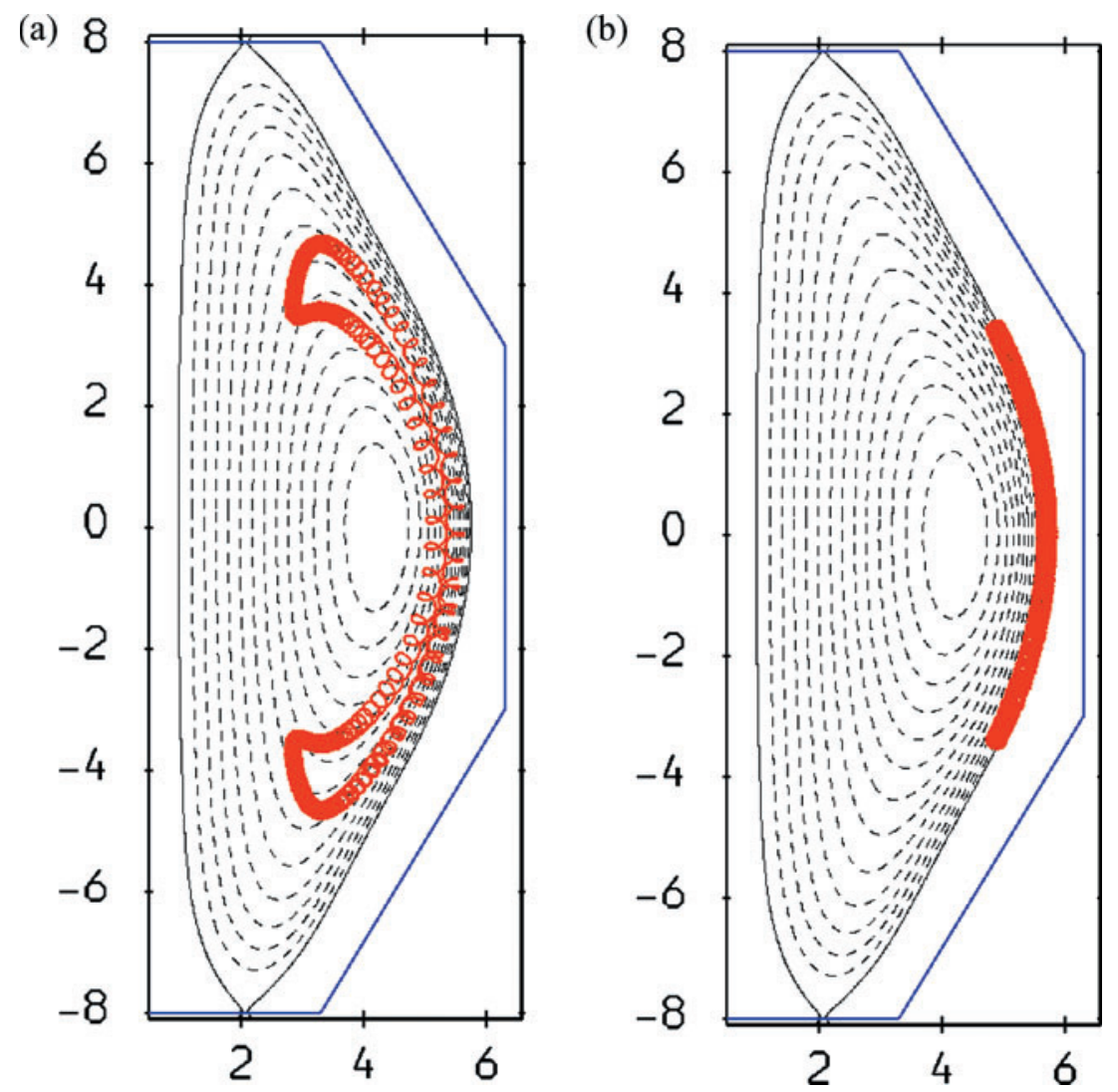

Figure 13. Trapped 3.5 Mev $\alpha$-particle orbits in the STPP equilibrium (a) for a particle born between the centre and the edge and $(b)$ for one born at the edge.

To calculate the lost $\alpha$-particle fraction, we compute the trajectories of $3.5 \mathrm{MeV} \alpha$-particles distributed uniformly in pitch angle at their birth and distributed in real space according to the predicted fusion production rate (assuming that this rate is dominated by reactions between thermal ions). CUEBIT then follows the orbits for one slowing down time (evaluated close to the plasma edge). A particle is considered to be lost if it strikes the vacuum vessel wall. We find that the loss rate, approximately $1 \%$, is acceptably low.

\subsection{Fuelling}

We now address the steady-state fuelling for the plasma. Our starting point is the global particle balance equation, derived by integrating the continuity equation over the plasma volume:

$$
\frac{\mathrm{d} N}{\mathrm{~d} t}+\frac{N}{\tau_{\mathrm{p}}}=\Phi_{\mathrm{G}}+\Phi_{\mathrm{NB}}
$$

Here, $N$ is the total particle inventory, $\tau_{\mathrm{p}}$ the particle confinement time, $\Phi_{\mathrm{G}}$ the particle source rate from either gas 
fuelling (continuous) or pellet fuelling (discrete) and $\Phi_{\mathrm{NB}}$ is the neutral beam source rate. Assuming the particle confinement time to be independent of time, equation (9) can be integrated, with the solution

$N(t)=N_{0} \mathrm{e}^{-t / \tau_{\mathrm{p}}}+\left(1-\mathrm{e}^{-t / \tau_{\mathrm{p}}}\right) \tau_{\mathrm{p}} \Phi_{\mathrm{NB}}+\mathrm{e}^{-t / \tau_{\mathrm{p}}} \int_{0}^{t} \Phi_{\mathrm{G}}\left(t^{\prime}\right) \mathrm{e}^{t^{\prime} / \tau_{\mathrm{p}}} \mathrm{d} t^{\prime}$,

where a constant fuelling rate from the NBI has been assumed and $N_{0}$ is the particle density at time $t=0$. For either gas fuelling, or a situation where many pellets are injected within a particle confinement time, the time-dependence of $\Phi_{\mathrm{G}}$ can be neglected, and the solution is simplified:

$$
N(t)=N_{0} \mathrm{e}^{-t / \tau_{\mathrm{p}}}+\tau_{\mathrm{p}}\left(\Phi_{\mathrm{NB}}+\Phi_{\mathrm{G}}\right)\left(1-\mathrm{e}^{-t / \tau_{\mathrm{p}}}\right) .
$$

To derive the fuelling rate from the NBI, we simply use the results from section 3.3 and assume that all the current drive is provided by NBI. The fuelling rate is given by the power divided by the energy, $\Phi_{\mathrm{NB}}=3.4 \times 10^{21} \mathrm{~s}^{-1}$, which is dominated by the low energy beam system. In the limit $t \rightarrow \infty$ the required number of fuel atoms is given by the product of the volume averaged density $\left(1.08 \times 10^{20} \mathrm{~m}^{-3}\right)$ and the plasma volume $\left(1175 \mathrm{~m}^{3}\right)$, yielding $1.27 \times 10^{23}$. Unless the particle confinement time is very large (i.e. $\tau_{\mathrm{p}}=37 \mathrm{~s}=20 \tau_{E}$ ), the neutral beam fuelling rate is insufficient, and additional fuelling must be provided.

To be definite, let us adopt a particle confinement time consistent with that assumed for the helium ash removal, i.e. $\tau_{\mathrm{p}}=8 \mathrm{~s}=4 \tau_{E}$ (see table 1 ). A steady fuelling rate of $\Phi_{\mathrm{G}}=1.25 \times 10^{22} \mathrm{~s}^{-1}$ is required from an additional fuelling system. Gas puffing at the plasma edge is a possible option, but if pellet injection is viable, it may be a more efficient fuelling method and may allow a degree of control over the density profile. In the remainder of this sub-section we make estimates for the requirements of a pellet injection system.

We first estimate the pellet penetration length, $\lambda$. A model which agrees reasonably well with an international pellet ablation database is the neutral gas shielding (NGS) model (although it should be noted that the database consisted of lower temperature discharges than that modelled here, and the effects of energetic $\alpha$-particles are not taken into account). The NGS model provides the following estimate [41]:

$$
\frac{\lambda}{a}=0.79 T_{\mathrm{e}}^{-5 / 9} n_{\mathrm{e}}^{-1 / 9} N_{\mathrm{pel}}^{5 / 27} v_{\mathrm{pel}}^{1 / 3},
$$

where $a$ is the plasma minor radius, $T_{\mathrm{e}}$ and $n_{\mathrm{e}}$ are the central electron temperature (in $\mathrm{keV}$ ) and density (in $10^{20} \mathrm{~m}^{-3}$ ), respectively, $N_{\mathrm{pel}}$ is the number of atoms in the pellet (in units of $10^{20}$ ) and $v_{\text {pel }}$ is the pellet velocity (in $\mathrm{km} \mathrm{s}^{-1}$ ). Defining a pellet injection frequency, $v$, and using the required fuelling rate derived above, we have $N_{\text {pel }}=125 / \nu$. With the STPP parameters $T_{\mathrm{e}}=30$ and $n_{\mathrm{e}}=1.08$, we then have

$$
\frac{\lambda}{a}=0.29 \frac{v_{\mathrm{pel}}^{1 / 3}}{v^{5 / 27}}
$$

so that a low pellet injection frequency (i.e. larger pellet) and high injection velocity will result in a deeper penetration.

Let us estimate the pellet requirements to fuel at the magnetic axis. The Shafranov shift of the STPP equilibrium

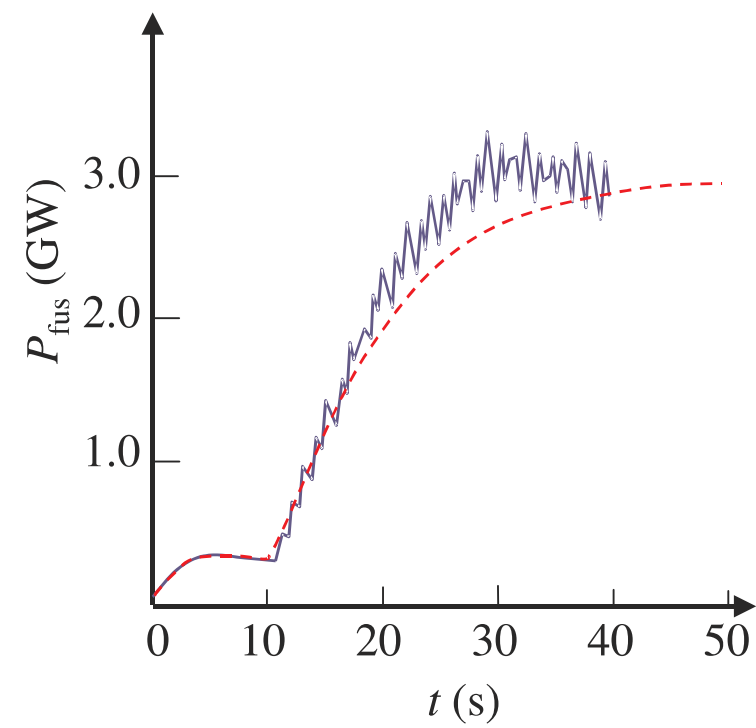

Figure 14. The calculated evolution of fusion power assuming a constant fuelling process (- - - ) and for pellet injection (- - at a rate of $1 \mathrm{~s}^{-1}$. The pellet properties are described in the text.

is very large, so that the magnetic axis is at a major radius $R_{0}=4.64 \mathrm{~m}$. This significantly reduces the distance that the pellet must travel so that, for central fuelling from the outboard midplane, $\lambda / a=0.5$. To avoid large fluctuations in the fusion power, which would occur if the pellet injection frequency were low, we require $\nu \tau_{\mathrm{p}} \gg 1$. To give a definite example, we take $v=1 \mathrm{~s}^{-1}$, in which case, we require a pellet velocity of $v_{\text {pel }}=5.1 \mathrm{~km} \mathrm{~s}^{-1}$. The number of atoms in the pellet is then $N_{\text {pel }}=125$, corresponding to a pellet radius $\sim 6-7 \mathrm{~mm}$. This pellet velocity is rather high, but it can be reduced to less than $1 \mathrm{~km} \mathrm{~s}^{-1}$ if it is sufficient to penetrate half way to the magnetic axis.

In figure 14 we show the fusion power that would result from the solution for the number of particles given by equation (10). Two cases are considered, both of which assume $\tau_{\mathrm{p}}=8 \mathrm{~s}$. In the first we show how the fusion power evolves if a continuous fuelling process is used, reaching a steadystate level in $\sim 50 \mathrm{~s}$. The second case shows the evolution of fusion power with pellet injection, assuming the pellet properties derived above. The pellet is assumed to ablate over a time $0.2 \mathrm{~ms}$.

The database on which equation (12) was validated was provided by conventional tokamaks [41], and its relevance to STs is uncertain. One issue, which may be particularly important for the STPP equilibrium, is related to the magnetic geometry. In conventional tokamaks, pellet injection is found to be more effective from the inboard side [42], in which case the magnetic field is decreasing along the path of the pellet. In the STPP equilibrium, however, the magnetic field will decrease along the pellet trajectory even if the pellet is injected from the outboard side (see figure 8). Thus, it is possible that pellet fuelling may be more efficient than the estimates we have provided here, which do not include this effect. Twodimensional modelling of the pellet ablation process, together with further experiments on STs are required to make more precise calculations about the requirements for pellet injection, but it clearly appears possible that such a scheme is viable for STPP. 
Table 2. The eight best fitting SOL width, $\Delta_{\mathrm{h}}$, scalings for the collisional SOL cases, together with a brief description of the physical basis for each of the models. $L_{p}, L_{n}$ and $L_{T}$ are the perpendicular gradient lengths for pressure, density and temperature contained in some of the theoretical expressions for the cross-field thermal diffusivity, $\chi_{\perp}$.

\begin{tabular}{lll}
\hline Model [44] & Physics basis for $\chi_{\perp}$ & $\Delta_{h}$ scaling \\
\hline E1-II & Resistive MHD interchange: with $L_{p}$ & $\Delta_{\mathrm{h}} \propto n^{14 / 15} R_{\mathrm{g}}^{9 / 16} B^{-14 / 15} L_{\mathrm{c}}^{16 / 15} P_{\text {surf }}^{-2 / 5}$ \\
E2-II & Resistive MHD interchange: with $L_{n} \sim L_{T}$ & $\Delta_{\mathrm{h}} \propto n^{14 / 15} R_{\mathrm{g}}^{9 / 16} B^{-14 / 15} L_{\mathrm{c}}^{16 / 15} P_{\text {surf }}^{-2 / 5}$ \\
H-II & with $L_{n} \sim L_{T}$ & $\Delta_{\mathrm{h}} \propto n^{7 / 9} B^{-7 / 12} L_{\mathrm{c}}^{5 / 6} P_{\text {surf }}^{-13 / 36}$ \\
N-II & Bohm & $\Delta_{\mathrm{h}} \propto n^{7 / 11} B^{-7 / 11} L_{\mathrm{c}}^{8 / 11} P_{\text {surf }}^{-3 / 11}$ \\
E2-III & Resistive MHD interchange: with $L_{n}$ & $\Delta_{\mathrm{h}} \propto n^{35 / 26} R_{\mathrm{g}}^{17 / 31} B^{-14 / 13} L_{\mathrm{c}}^{31 / 26} P_{\text {surf }}^{-8 / 13}$ \\
K2-II & Drift Alfven: higher collisionality & $\Delta_{\mathrm{h}} \propto n^{49 / 78} B^{-28 / 39} L_{\mathrm{c}}^{51 / 78} P_{\text {surf }}^{-10 / 39}$ \\
F-III & Drift: with $L_{n}$ & $\Delta_{\mathrm{h}} \propto n^{5 / 8} B^{-3 / 4} L_{\mathrm{c}}^{5 / 8} P_{\text {surf }}^{-1 / 4}$ \\
Q-II & Constant & $\Delta_{\mathrm{h}} \propto n^{7 / 9} L_{\mathrm{c}}^{4 / 9} P_{\text {surf }}^{-5 / 9}$ \\
\hline
\end{tabular}

Table 3. Predictions for the STPP mid-plane SOL widths derived from the models of table 2, together with the average of the models.

\begin{tabular}{lllllllll}
\hline Models & E1/E2-II & H-II & N-II & E2-III & K2-II & F-III & Q-II & Average \\
\hline$\Delta_{\mathrm{h}}^{\text {outer }}(\mathrm{mm})$ & 44.2 & 22.3 & 19.3 & 40.4 & 16.3 & 15.2 & 18.0 & $25 \pm 12$ \\
$\Delta_{\mathrm{h}}^{\text {inner }}(\mathrm{mm})$ & 57.3 & 36.8 & 23.0 & 66.2 & 15.5 & 13.3 & 65.8 & $40 \pm 23$ \\
\hline
\end{tabular}

\subsection{Exhaust}

The final topic, and one of the most challenging, is the exhaust. The compact nature of the device, while being attractive from an economic point of view, could lead to high power densities in the exhaust. To help alleviate the problem we operate in a double null configuration. Apart from the obvious benefit that this doubles the available target area over which we can spread the scrape-off layer (SOL) heat load, there is the additional advantage that most of the power is then expected to go to the outboard divertor target plates. This is important because the small major radius of the inner target plates leads to a very small area on which to handle the heat load. There are a number of reasons why one would expect the majority of the heat to go to the outboard side: the outboard cross-sectional area of the plasma is larger; the turbulence responsible for driving the anomalous transport is likely to have an outboard-ballooning nature, and the Shafranov shift leads to a compression of the flux surfaces on the outboard side. Indeed, on MAST something in the region of $95 \%$ of the power flows to the outboard side (although this varies, depending on the conditions) [43]. Thus, we assume that in the power plant there will be a similar split between the inboard and outboard divertors. In addition, we assume that $50 \%$ of the power can be radiated.

To evaluate the power loading at the target plates, we require an estimate of the midplane SOL width, $\Delta_{\mathrm{h}}$. A number of theoretical models [44] have been assessed by comparing with the MAST L-mode data, and eight 'best fit' models survive (see table 2) [45]. In the expressions for $\Delta_{h}$, we have defined the power per unit area across the separatrix, $P_{\text {surf }}$, and the parallel connection length, $L_{\mathrm{c}}$, is taken half way across the SOL. The density, $n$, is the line-averaged value, the magnetic field, $B$, is evaluated at the target. We evaluate the outer and inner SOL widths for STPP independently. The parameters are: power across separatrix $=340 \mathrm{MW}(18 \mathrm{MW})$, $B=1.3 \mathrm{~T}(6.0 \mathrm{~T}), L_{\mathrm{c}}=50 \mathrm{~m}(140 \mathrm{~m})$ and the surface area of the separatrix is $490 \mathrm{~m}^{2}\left(120 \mathrm{~m}^{2}\right)$ for the outer (inner) target. The resulting estimates for $\Delta_{\mathrm{h}}$ are given in table 3 .

The SOL widths at the target plates can be broader than those at the midplane due to the poloidal flux expansion, $f$.
This is estimated from the free-boundary equilibrium to be $f \sim 3.5$ on the outer target and $f \sim 1$ on the inner target. Adopting target plates angled at $10^{\circ}$, the heat flux at the target plate can then be estimated. Employing the SOL widths derived from averaging all models, we find heat loads of $17.8 \pm 8.5 \mathrm{MW} \mathrm{m}^{-2}$ for the outer target and $4.4 \pm 2.5 \mathrm{MW} \mathrm{m}^{-2}$ for the inner target. However, there is a wide range of SOL widths predicted by the 'acceptable' models listed in table 2 . Taking the worst case, we find that the outer target load increases to $29.3 \mathrm{MW} \mathrm{m}^{-2}$, while the inner target 'worst case' is $13.2 \mathrm{MW} \mathrm{m}^{-2}$.

Although there is a wide range of predictions from acceptable models for the SOL width, and the extrapolation uncertainties are therefore large, it is unlikely that the continuous exhaust can be handled by conventional materials, since the erosion rates are expected to require frequent replacement of target surfaces. In addition, we have not attempted to address the challenging task of estimating the power loads from transient events, such as edge-localized modes (ELMs) and possible disruptions. Uncertainties in exhaust power loads need to be reduced by improved models and extended databases. Nevertheless, it seems highly likely that conventional exhaust-handling scenarios will be inadequate and it is therefore important to continue to develop novel exhaust-handling schemes, such as the cascading pebble divertor [8], in which the target surface is continuously replaced.

\section{Conclusions}

In this paper, we have considered many of the key plasma physics issues associated with the steady-state operation of a fusion power plant based on the ST concept. While there remain a number of uncertainties, we have demonstrated that a self-consistent solution does exist, and that the ST remains a strong contender for ultimately providing fusion power.

In our discussion of the various plasma physics issues, we have identified a number of advantages associated with steady-state plasma operational scenarios that the ST may 
be able to offer. Furthermore, technological benefits $[7,8]$, mainly associated with the simplicity of the design and ease of maintenance, make the ST an attractive option for providing fusion energy.

Of course, there remain some major challenges to meet before we can claim to have fully demonstrated the viability of a ST fusion power plant. For example, we have only concerned ourselves here with the final, steady-state solution and not addressed how the plasma might be initiated without the use of a central solenoid. While there are a number of ideas for non-inductive start-up scenarios, supplemented by an inductive component from ramping the poloidal field coil currents, these have yet to be fully developed. Key issues associated with the sustainment of the plasma include the role of fast particle instabilities, and whether there is sufficient current profile control to optimize confinement and avoid MHD instabilities. Indeed, it is necessary to characterize the confinement properties of such high $\beta$ plasmas, with a hollow current profile (yet monotonic $q$ profile) to determine, for example, whether or not $H=1.6$ is achievable. We also need to assess our ability to provide off-axis non-inductive current drive, and experiments are required to validate models for both neutral beam and RF (e.g. EBW) current drive schemes. An assessment of the role of flow (e.g. for stabilizing resistive wall modes or its impact on confinement), and the ability to impart momentum in the plasma (e.g. by the off-axis beams) will require further study. Again, associated with the sustainment is the issue of fuelling, and how the non-monotonic variation of the magnetic field with major radius influences pelletinjection and the resulting density profile, for example. On issues associated with the exhaust, it is encouraging that the steady-state heat loads do appear to be manageable, but the extrapolation uncertainties are large, and ongoing research should aim to minimize these uncertainties. The impact of transients such as ELMs and disruptions (if they are present) also needs to be addressed before the exhaust characteristics can be fully quantified. Finally, associated with the exhaust, is the issue of helium ash and whether this can be removed effectively in the absence of sawteeth.

To summarize, there has been much progress in understanding the plasma physics processes that govern the performance of STs. These advances have strengthened the basis for the proposal that such devices can provide an attractive solution for generating fusion energy. Nevertheless, there remain a number of crucial questions to be tackled to demonstrate fully the viability of a ST fusion power plant.

\section{Acknowledgments}

This work was funded jointly by the UK Engineering and Physical Sciences Research Council and by EURATOM.

\section{References}

[1] Peng Y.-K.M. and Hicks J.B. 1990 Proc. 16th Symp. on Fusion Technology (London: Elsevier) p 1287

[2] Hender T.C. et al 1993 Proc. 14th Int. Conf. on Plasma Physics and Controlled Nuclear Fusion Research 1992 (Würzburg, 1992) vol 3 (Vienna: IAEA) p 399

[3] Buttery R. et al 1995 Proc. 15th Int. Conf. on Plasma Physics and Controlled Nuclear Fusion Research 1994 (Seville, 1994) vol 2 (Vienna: IAEA) p 633
[4] Peng Y.-K.M. et al 1995 Proc. 15th Int. Conf. on Plasma Physics and Controlled Nuclear Fusion Research 1994 (Seville, 1994) vol 2 (Vienna: IAEA) p 643

[5] Stambaugh R.D., Chan V.S., Miller R.L. and Schaffer M.J. 1998 Fusion Technol. 33

[6] Robinson D.C. et al 1998 Proc. 17th Int. Conf. on Fusion Energy (Yokohama, 1998) (Vienna: IAEA) CD-ROM file FTP/05 and http://www.iaea.org/programmes/ripc/physics/ start.htm

[7] Voss G.M. et al 2000 Fusion Eng. Des. B 51309

[8] Voss G.M., Bond A., Hicks J.B. and Wilson H.R. 2002 Fusion Eng. Des. 6365

[9] F. Najmabadi and the ARIES Team 2003 Fusion Eng. Des. 65143

[10] Jardin S.C. et al 2003 Fusion Eng. Des. 65165

[11] Miller R.L. and the ARIES Team 2003 Fusion Eng. Des. 65199

[12] Akers R.J. et al 2000 Nucl. Fusion 401223

[13] Wilson H.R. et al 2002 Proc. 19th Int. Conf. on Fusion Energy 2002 (Lyon, 2002) (Vienna: IAEA) CD-ROM file FT/1-5 and http://www.iaea.org/programmes/ripc/physics/fec2002/ $\mathrm{html} / \mathrm{fec} 2002 . \mathrm{htm}$

[14] Darke A.C. et al 1995 The mega ampere spherical tokamak Proc. 16th Symp. on Fusion Engineering (IEEE/NPSS) (Champaign-Urbana, Illinois)

[15] Akers R.J. et al 2003 Plasma Phys. Control. Fusion 45 A175

[16] Wong C.P.C. and Stambaugh R.D. 2000 Fusion Eng. Des. 51-52 387

[17] Miller R.L. et al 1997 Phys. Plasmas 41062

[18] ITER Physics Basis 1999 Nucl. Fusion 392175

[19] Kotschenreuther M., Rewoldt G. and Tang W.M. 1995 Comput. Phys. Commun. 88128

[20] Knight P.J., Cox M. and Hender T.C. 1992 Comput. Phys. Commun. 7199

[21] Leuer J.A. 1989 Fusion Technol. 15489

[22] Mercier C. 1960 Nucl. Fusion 147

[23] Hole M.J. et al 2004 Ideal MHD stability of the mega ampere spherical tokamak and the spherical tokamak power plant, in preparation

[24] Wilson H.R. 2002 Trans. Fusion Sci. Technol. 41107

[25] Sauter O. et al 1997 Phys. Plasmas 41654

[26] Buttery R.J. et al 2002 Phys. Rev. Lett. 88125005

[27] Hegna C.C. 1999 Phys. Plasmas 63980

[28] Akers R.J. et al 2002 Nucl. Fusion 42122

[29] Hawyluk R.J. 1980 Physics of Plasmas Close to Thermonuclear Conditions vol 1, ed B. Coppi et al (Brussels: CEC) p 19

[30] O'Brien M. et al 1993 Proc. IAEA TCM on Advanced Simulations and Modelling of Thermonuclear Plasma (Montreal, 1992) (Vienna: IAEA) p 527

[31] Preinhaelter J. and Kopecky V. 1973 J. Plasma Phys. 101

[32] Fisch N.J. and Boozer A.H. 1980 Phys. Rev. Lett. 24720

[33] Cairns R.A., Owen J. and Lashmore-Davies C.N. 1983 Phys. Fluids 263475

[34] Ohkawa T. 1976 General Atomics Report no GA-A13847

[35] ITPA H-mode Power Threshold Database Working Group (presented by Takizuka T.) 2004 Plasma Phys. Control. Fusion 46 A227

[36] Pereverzev G.V. and Yushmanov P.N. 2002 IPP Report 5/98

[37] Dnestrovskij Yu.N. et al 2004 Plasma Phys. Rep. 301

[38] Kotschenreuther M. et al 2000 Nucl. Fusion 40677

[39] Hahm T.S. and Burrell K. 1995 Phys. Plasmas 21648

[40] Hamilton B., McClements K.G., Fletcher L. and Thyagaraja A. 2003 Sol. Phys. 214339

[41] Baylor L.R. et al 1997 Nucl. Fusion 37445

[42] Lang P.T. et al 1997 Phys. Rev. Lett. 791487

[43] Counsell G.F. et al 2002 Plasma Phys. Control. Fusion 44827

[44] Connor J.W. et al 1999 Nucl. Fusion 39169

[45] Ahn J.-W. et al 2004 L-mode SOL width scaling in the MAST spherical tokamak and extrapolations to the future machine Plasma Phys. Control. Fusion submitted 